Paediatr Paedolog 2018 53:1-3 https://doi.org/10.1007/s00608-017-0544-3 Online publiziert: 9 . Januar 2018 (c) Springer-Verlag GmbH Austria, ein Teil von Springer Nature 2018

CrossMark

\section{Daniela Karall}

Department für Kinder- und Jugendheilkunde, Universitätsklinik für Pädiatrie I, Bereich Angeborene Stoffwechselstörungen, Medizinische Universität Innsbruck, Innsbruck, Österreich

\title{
Neue Präsidentschaftsperiode für die Österreichische Gesellschaft für Kinder- und Jugendheilkunde
}

Es ist mir eine Ehre und Freude, zum Januar 2018 von Univ.-Prof. Dr. Wolfgang Sperl die Präsidentschaft der Österreichischen Gesellschaft für Kinder- und Jugendheilkunde (ÖGKJ) zu übernehmen!

Erstmalig in der 55-jährigen $\mathrm{Ge}$ schichte der Österreichischen Gesellschaft für Kinder- und Jugendheilkunde ist nun eine Frau in der Position der Präsidentschaft - somit vielleicht so etwas wie eine neue Ära bzw. das Einschwenken in die Entwicklungen der aktuellen Zeit.

\section{Biografie und Schwerpunkte}

Meine Kindheit und Jugend verbrachte ich großteils in Mexico City; Medizin studierte ich in Innsbruck, wo ich auch - bis auf einen 15-monatigen Forschungsaufenthalt in Melbourne in Australien - die Facharztausbildung Kinderund Jugendheilkunde absolvierte. Mein erster Schwerpunkt war von Anfang an die Betreuung von Kindern, Jugendlichen und jungen Erwachsenen mit angeborenen Stoffwechselstörungen und damit seltenen Krankheiten. Da die Therapie bei vielen dieser Erkrankungen im Wesentlichen eine Ernährungsmodifikation, also eine Ernährungstherapie, darstellt, habe ich mich bald auch mit Ernährung beschäftigt. In der Pädiatrie stehen wir begleitend ganz am Anfang des Lebens und legen dort essenzielle Weichen für das ganze Leben von Menschen. Ich bin davon überzeugt, dass Impfwesen und die gute Etablierung einer sinnvollen, altersentsprechenden Ernährung (Stichwort Förderung von Stillen) die beiden Säulen sind, die uns in der Prävention von Erkrankungen weltweit in puncto Erhöhung der Lebenserwartung und Verbesserung der Lebensqualität durch Reduktion von Mortalität und Morbidität am meisten gebracht haben und bringen werden.

Somit beinhaltet mein persönlicher Werdegang die zwei Schwerpunktthemen angeborene Stoffwechselstörungen und Ernährung des Säuglings und Kindes, die in meinen Augen gut die Dichotomie innerhalb der ÖGKJ widerspiegeln, die den Bogen spannt zwischen Seltenem und Häufigem, also einerseits der hochspezialisierten Medizin und andererseits der primären (präventiven) Versorgung der Altersgruppe der 0- bis 18-Jährigen. Beide Bereiche sind integraler Bestandteil der Agenden der ÖGKJ und für mich wie die beiden Seiten einer Münze, die sehr unterschiedlich sein können und trotzdem Teil des Ganzen sind.

\section{Vernetzung ist wichtig}

Ich persönlich profitierte immer von der engen nationalen (und auch internationalen) Vernetzung zwischen Gleichgesinnten und bin überzeugt, dass Vernetzung wertvolle Ausrüstung für eine bestmögliche Versorgung von Patienten ist. Der fachliche und persönliche Austausch ist das geeignete Mittel, mit dem wir den zum Teil sehr unterschiedlichen Bedürfnissen von Kindern, Jugendlichen und deren Eltern und Familien am besten begegnen. Bis heute bin ich gern in der Pädiatrie tätig und ich würde diese Fachdisziplin gegen keine andere tauschen!

\section{Die Österreichische Ge- sellschaft für Kinder- und Jugendheilkunde (ÖGKJ)}

Die Österreichische Gesellschaft für Kinder- und Jugendheilkunde (ÖGKJ) als Fachvertretung und offizieller $\mathrm{Zu}$ sammenschluss des Berufsverbands ist keine kleine Gesellschaft - kein kleiner Haufen - mehr: Sie hat inzwischen über 1600 Mitglieder. Die Vernetzung über die ÖGKJ ist in diesem Sinn nicht zu unterschätzen. Auch wenn der persönliche Mehrwert unter Umständen nicht sofort ersichtlich ist, möchte ich doch dazu ermutigen, sich der Gesellschaft anzuschließen. Sie ist in ihrer Struktur über mehrere Jahrzehnte gewachsen. So kann auf bewährten Strukturen aufund weitergebaut werden, was ich sehr schätze. Jede(r) Einzelne hat etwas beizutragen - gemeinsam sind wir auf jeden Fall sichtbarer!

\section{Agenda}

Bestehende Agenden werden in den nächsten drei Jahren weiter verfolgt werden, wie $z$. B.

- Präsenz, Beratung, Ansprechpartnerschaft in Fragen der Kinderund Jugendgesundheit für die Bundesministerien für Gesundheit und Soziales und den Hauptverband der Sozialversicherungsträger;

- adäquate Positionierung der Pädiatrie in Ausbildungsfragen;

- Sicherstellung der Spezialisierungen in der Pädiatrie; 
- Ausrollung der Rehabilitation für Kinder und Jugendliche in Österreich;

- Vernetzung mit verwandten Gesellschaften wie der Deutschen Gesellschaft für Kinder- und Jugendmedizin (DGKJ);

- weitere Einbeziehung, unter anderem der politischen Kindermedizin und der Kinderliga.

Dabei ist sehr wertvoll und hilfreich, dass die ÖGKJ mit Beginn 2018 mit unserem geschätzten Kollegen Prim. Univ.Prof. Dr. Reinhold Kerbl (Leiter der Pädiatrie in Leoben) über einen Geschäftsführer verfügt, der über die laufenden Agenden einen Bogen der Kontinuität spannen wird.

\section{Besonders wichtige Bereiche}

Für die nächsten drei Jahre stehen drei Bereiche auf der Agenda, denen ich mich gemeinsam mit meiner geschätzten Kollegin PD Dr. Scholl-Bürgi (ÖGKJ-Sekretärin für die nächsten 3 Jahre) besonders widmen möchte.

\section{Nachwuchsförderung}

Der Aspekt der guten Ausbildung und Ausrüstung von Nachfolgern in allen Bereichen der Pädiatrie betrifft selbstverständlich nicht nur unser Fach, sondern zunehmend auch andere Disziplinen. Allerdings ist die Pädiatrie insofern besonders, da sie eigentlich und zunehmend ein weibliches Fach ist (hoher Frauenanteil) und daher der Bedarf an Teilzeitmodellen sowohl für die Ausbildungszeit als auch für die spätere Berufstätigkeit möglicherweise schon früher als in anderen Fächern sichtbar geworden ist. Zunehmend sind wir herausgefordert, die Aspekte, die die jüngere Generation in die medizinische Versorgung hereinbringt, in die Zukunftsplanung des Fachs mit einzubauen. Ich erachte Schlagwörter wie Generation Y, Work-Life-Balance nicht nur als Herausforderung, sondern insbesondere auch als Chance, Modelle zu erarbeiten, die zukünftig und langfristig eine tragfähige medizinische Versorgung von Kindern und Jugendlichen möglichst wohnortnah und flächendeckend gewährleisten.

\section{Öffentlichkeitsarbeit}

Öffentlichkeitsarbeit ist für die ÖGKJ nichts Neues, sondern schon lange essenzieller Bestandteil innerhalb der Gesellschaft (aktuell: ÖGKJ-Pressestelle bei Ralph Kerschbaumer, studio12). Allerdings sind wir als in der Medizin Tätige meist nicht damit vertraut, Medien sinnvoll zu nutzen, um unsere Inhalte zu transportieren. Der Trend der Zeit fordert uns, dass wir uns mit den medialen Möglichkeiten auseinandersetzen und sie positiv anwenden. Ziel für mich wäre es, eine Dynamik innerhalb der ÖGKJ zu entwickeln bzw. zu erreichen, in der die Mitglieder für sie wichtige und verschiedene Themen einbringen, die wir als Gesellschaft unterstützen und verbreiten können.

\section{Vernetzung}

Vernetzung ist für mich das Stichwort für eine funktionierende, sich entwickelnde Gemeinschaft (mit Reden kommen die Leut' zusammen). In erster Linie gilt die Vernetzung zwischen Kollegen, übergreifend das Alter und die Spezialisierung betreffend. Am besten wird einfach von Vorbildern (Mentoring) gelernt, sowohl hinsichtlich der klinischen Tätigkeit als auch der wissenschaftlichen Arbeit. Für das Weiterkommen, Projekt-Förderung und ähnliches ist die Einbindung in eine Gruppe bzw. ein Netzwerk nicht mehr wegzudenken. In diesem Sinn wünsche ich mir, dass es uns gelingt, den guten Austausch und die bereits bestehenden Netzwerke über die ÖGKJ noch besser sichtbar und damit effektiver $\mathrm{zu}$ machen, sowohl was die klinische Tätigkeit als auch die wissenschaftliche Arbeit betrifft. Ein Instrument für Austausch und Vernetzung könnte vermehrt auch die ÖGKJ-Homepage werden, die beginnend in den letzten beiden Jahren eines Facelifts unterzogen wurde, mit dem sie im neuen Jahr online geht (www. paediatrie.at).

\section{Schlussworte}

Abschließend möchte ich betonen, dass wir in Österreich aufgrund der überschaubareren Größe unseres Landes einen Vorteil durch gute Vernetzung genießen. Nicht nur im Bereich der spe-

zialisierten Medizin, sondern auch im Rahmen der einzelnen Disziplinen und Bereiche der Pädiatrie kennen die Player sich untereinander und sind regional, national sowie auch international gut miteinander vernetzt. Das ist eine sehr gute Voraussetzung für die State-of-theArt-Betreuung von Patienten.

Gern nehme ich Ideen, Vorschläge und Pläne für die nächste Periode auf und zähle weiterhin auf Unterstützung und gute Zusammenarbeit!

Der Jahreswechsel ist immer eine gute Gelegenheit, Rückblick zu halten, Bilanz zu ziehen, um mit neuem Schwung zweite Chancen zu ergreifen, sich bestehenden und neuen Herausforderungen zu stellen und mit freudiger Spannung Kommendes zu erwarten!

In diesem Sinn wünsche ich einen erfolgreichen Start in ein segensreiches neues Jahr 2018!

Ihre/Eure

Daniela Karall

\section{Korrespondenzadresse

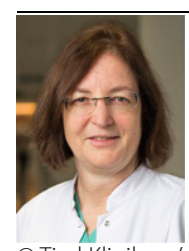 \\ () Tirol Kliniken \\ Medizinische Universität \\ Innsbruck \\ Ao. Univ.-Prof. Dr. D. Karall, IBCLC \\ Department für Kinder- und Jugendheilkunde, Universitätsklinik für Pädia- trie I, Bereich Angeborene Stoffwechselstörungen, Medizinische Universität Innsbruck \\ Anichstraße 35, 6020 Inns- bruck, Österreich daniela.karall@i-med.ac.at}

Ao. Univ.-Prof. Dr. Daniela Karall Seit Januar 2018 Präsidentin der Österreichischen Gesellschaft für Kinder- und Jugendheilkunde; Stellvertretende Direktorin der Klinik für Pädiatrie I, Department Kinderund Jugendheilkunde, Medizinische Universität Innsbruck; Bereich Angeborene Stoffwechselstörungen; Innsbruck, Österreich.

Interessenkonflikt. D. Karall gibt an, dass kein Interessenkonflikt besteht. 
Hier steht eine Anzeige.

\section{曾 Springer}

